\title{
Remarks on Mohrhoff's Interpretation of Quantum Mechanics
}

\author{
Louis Marchildon \\ Département de physique, Université du Québec, \\ Trois-Rivières, Qc. Canada G9A 5H7 \\ email: marchild@uqtr.ca
}

\begin{abstract}
In a recently proposed interpretation of quantum mechanics, $\mathrm{U}$. Mohrhoff advocates original and thought-provoking views on space and time, the definition of macroscopic objects, and the meaning of probability statements. The interpretation also addresses a number of questions about factual events and the nature of reality. The purpose of this note is to examine several issues raised by Mohrhoff's interpretation, and to assess whether it helps providing solutions to the long-standing problems of quantum mechanics.
\end{abstract}

KEY WORDS: quantum mechanics; interpretation; Mohrhoff.

\section{Introduction}

More than 75 years after quantum mechanics was proposed and the wave function given a probabilistic interpretation, the issue of interpreting the theory is anything but settled. The past 25 years have witnessed, among other avenues, the revival of Bohmian mechanics [1, 2, 3, the development of decoherence theory [4, the consistent histories approach [5, 6], spontaneous localization theories [7], the evolution of the modal interpretation [8, 9], and the idea of a veiled reality [10].

The main question all these approaches try to answer is the following: How can one reconcile the apparently indeterminate nature of quantum observables with the apparently determinate nature of classical observables, if 
the latter are reduced to the former? The question shows up most spectacularly in the so-called quantum measurement problem, recognized early after the advent of quantum mechanics [1]. It is also deeply connected with the meaning of probability statements.

A new and original way of looking at these problems has, in the past few years, been proposed by U. Mohrhoff. ${ }^{1}$ Like the Copenhagen interpretation, with which it shares a number of characteristics, it aims at "an interpretation of quantum mechanics that takes standard quantum mechanics to be fundamental and complete." [13, p. 6] But it goes beyond the Copenhagen interpretation in making rather striking proposals on several most fundamental issues, among them the structure of space-time, the nature of physical reality, and the meaning of objective probability.

This paper is a critical examination of a number of assertions made in Mohrhoff's interpretation, together with an analysis of their consequences. The various questions addressed here do not cover all aspects of the interpretation, which partakes of a wide-ranging system that reaches well into metaphysical ontology. The questions I investigate, however, carry much of the interpretation's specific answers to the problem of making sense of quantum mechanics.

Mohrhoff's interpretation of quantum mechanics is briefly summarized in the following section. Next I examine a number of issues it raises in connection with the Aharonov-Bergmann-Lebowitz (ABL) rule, the quantum state, and factual reality. I will try to assess whether the interpretation provides acceptable solutions to the problems of quantum mechanics, and the extent to which the proposed solutions are compulsory.

\section{Outline of Mohrhoff's interpretation}

It is impossible in just a few pages to really do justice to Mohrhoff's rich and complex interpretation. This section accordingly brings up those concepts and issues that subsequently will be the subject of analysis.

Central to Mohrhoff's interpretation is the belief, which he also finds in Mermin [18, "that all the mysteries of quantum mechanics can be reduced to the single puzzle posed by the existence of objective probabilities." [12, p. 728] As a matter of fact, the idea that understanding quantum mechanics requires

\footnotetext{
${ }^{1}$ Most relevant to the present discussion are Refs. 12, 13, 14, 15, 16, 17]. Others can be found in the e-print archives http://arXiv.org.
} 
understanding probability goes back at least to Popper [19], who for that purpose introduced his "propensity" interpretation of probability. Mohrhoff, however, views the problem differently. For him, objective probabilities must be assigned on the basis of all relevant facts, and this is done by the use of the Aharonov-Bergmann-Lebowitz rule [20].

The ABL rule can be formulated as follows. Let $A, C$, and $B$ be three observables, pertaining to a system $S$, which for our purposes can be taken as nondegenerate. At time $t_{1}, S$ is prepared in an eigenstate $|a\rangle$ of $A$. At time $t>t_{1}$, the observable $C$ is measured and one of the results $c_{j}$ is obtained. Finally, at time $t_{2}>t, B$ is measured and the result $b$ is found. Then the probability that $c_{i}$ is obtained at $t$, conditional upon the preparation of $|a\rangle$ at $t_{1}$ and the result $b$ at $t_{2}$, is given $b^{2}$

$$
P_{A B L}\left(c_{i}\right)=\frac{\left|\left\langle b \mid c_{i}\right\rangle\left\langle c_{i} \mid a\right\rangle\right|^{2}}{\sum_{j}\left|\left\langle b \mid c_{j}\right\rangle\left\langle c_{j} \mid a\right\rangle\right|^{2}} .
$$

Ket $|b\rangle$ is the eigenvector of $B$ associated with $b$ and the sum on $j$ runs over all eigenvalues of $C$. Here and elsewhere, all kets are assumed normalized.

How can the ABL rule be used to compute objective probabilities? According to Mohrhoff, probabilities of different outcomes are objective only if they are based on all relevant facts, past, present, and future. Thus if $C$ is measured, and the result $c_{i}$ is found, then the objective probability of $c_{i}$ is trivially one and the probability of $c_{j}$ for $j \neq i$ vanishes. Nontrivial objective probabilities can therefore only be assigned to measurements that are not performed. That is, they are assigned counterfactually. The ABL rule provides objective probabilities if it is interpreted as giving the probability of $c_{i}$ at $t$, on the conditions that (i) $S$ is prepared in $|a\rangle$ at $t_{1}$, (ii) no measurement is made between $t_{1}$ and $t_{2}$, and (iii) $S$ is found in $|b\rangle$ at $t_{2}$.

The Born probability of $c_{i}$ can also be defined as $\left|\left\langle c_{i} \mid a\right\rangle\right|^{2}$. But in the present context, it is subjective, inasmuch as it does not take into account the (later) result $b$. The Born probability is objective only if no measurement is performed on $S$ after its preparation at $t_{1}$.

In general, the ABL rule (as well as the Born rule if no measurement is performed after $t_{1}$ ) objectively assigns nontrivial probabilities to various possible results $c_{j}$. This, so the argument goes, has far-reaching consequences.

\footnotetext{
${ }^{2}$ With kets in the Schrödinger picture, the Hamiltonian is assumed to vanish between $t_{1}$ and $t_{2}$. Equation (11) also holds for a nonvanishing Hamiltonian, provided the Heisenberg picture is used instead.
} 
"The objectivity of quantum-mechanical probabilities [...] entails that the contingent properties of material objects are extrinsic rather than intrinsic." [12, p. 728] Extrinsic properties "supervene on what happens or is the case in the rest of the world." [15, p. 869] In between preparation and measurement or, more generally, outside the context of a property-indicating fact, no quantum observable has a value. This "nonvaluedness" holds also in the case where the objective probability of a given result is one. ${ }^{3}$ That is, one cannot assign an ontological "element of reality" to a quantum observable even if its objective (ABL or Born) probability is one. ${ }^{4}$

Facts, it turns out, are fundamental in Mohrhoff's interpretation. A typical fact (or matter of fact, actual event, or state of affairs) is the click of a Geiger counter. "Quantum mechanics always presupposes, and therefore never allows us to infer, the existence of a fact that indicates the alternative taken." [12, p. 733, original emphasized] Facts are uncaused and irreducible. Quantum mechanics can, for instance, give the subjective Born probability that $c_{i}$ is found at $t$ if $S$ is prepared at $t_{1}$ and if a $C$-indicating fact occurs at $t$. But it cannot predict, either categorically or probabilistically, that such a fact will indeed occur.

The genuine nonvaluedness of quantum observables applies in particular to space and time. Hence it should come as no surprise that "[t]he problem of understanding quantum mechanics is in large measure the problem of finding appropriate ways of thinking about the spatial and temporal aspects of the physical world." [14, p. 2] Space, for instance, has physical reality only if evinced by a position-indicating fact. In the two-slit experiment, the conceptual distinction we make between two regions of space does not exist for the electron unless a fact (e.g. a measurement successfully performed) indicates it. Such a fact will not in general indicate the position with arbitrary precision. The position of a quantum object therefore only exists when warranted by a fact, and then only within the limit where it is indeed war-

\footnotetext{
3 "Nonvaluedness" is introduced here with a meaning akin to Mohrhoff's "fuzziness." Both terms pertain to a quantum observable in between measurements. Fuzziness, however, specifically applies to the case where the objective probability of at least two results differs from zero.

${ }^{4}$ Elements of reality were introduced by Einstein, Podolsky, and Rosen in their seminal 1935 paper [21]. Following Mohrhoff, I use the characterization given by M. Redhead 22 p. 72]: "If we can predict with certainty, or at any rate with probability one, the result of measuring a physical quantity at time $t$, then at the time $t$ there exists an element of reality corresponding to the physical quantity and having a value equal to the predicted measurement result."
} 
ranted. "[T]he standard, substantival, set-theoretic conception of space is as inconsistent with quantum mechanics as absolute simultaneity is with special relativity." [14, p. 3] Space as an infinitely-differentiated objectively-existing manifold where events occur is simply a fiction. The same applies to time. Outside the context of a moment-indicating fact, time does not exist.

The nonexistence of time as an objective one-dimensional continuum, together with the nonvaluedness of all quantum observables outside the context of facts, leads Mohrhoff to conclude that there is no such thing as an evolving quantum state. The "state vector" $|\psi(t)\rangle$ (or the density operator $\rho(t)$ ) is strictly a tool used to compute either the subjective probability of various results at $t$ if a fact occurs at $t$, or the objective probability of results at $t$ if no fact occurs at $t$. It does not represent the time evolution of a state because (i) a state and a probability measure are logically different categories; (ii) there is no time $t$ if there is no fact at $t$; and (iii) no observable (even one which has $|\psi(t)\rangle$ as eigenvector) has a value unless the value is indicated by a fact. The last two reasons are related, for "[t]he insufficiency of Redhead's 'sufficiency condition' [for the existence of an element of reality] hinges on the nonexistence of the particular time $t$ in the absence of an actual measurement performed at the time $t$. [14, p. 23]

\section{The ABL rule}

The ABL rule was formulated in the previous section. It turns out that the rule can be interpreted in two very different ways. The two interpretations refer to different contexts, and as such are not mutually contradictory.

Suppose that $S$ (i) is prepared in $|a\rangle$ at $t_{1}$, (ii) undergoes a measurement of $C$ at $t$, and (iii) undergoes a measurement of $B$ at $t_{2}$ with the result $b$. Following the notation of Ref. [23, we shall call this an $A \rightarrow C \rightarrow B$ context. In that context, the $\mathrm{ABL}$ rule can be interpreted as giving the subjective probabilities of various results of the measurement of $C$. That the ABL rule means at least this much is uncontroversial.

Suppose now that $S$ (i) is prepared in $|a\rangle$ at $t_{1}$, (ii) undergoes no measurement between $t_{1}$ and $t_{2}$, and (iii) undergoes a measurement of $B$ at $t_{2}$ with the result $b$. This we shall call an $A \rightarrow B$ context. In that context, the ABL rule can be interpreted as asserting something at $t$ about an observable $C$ that has not been measured. This interpretation is controversial. It was not explicitly asserted by the original proponents of the rule [20, although 
one of them later held that they had indeed meant so [24].

What can be said in general about the second interpretation? From a purely logical point of view, it should be clear that one cannot, from uncontroversial factual properties of $C$ in an $A \rightarrow C \rightarrow B$ context, infer anything factual about $C$ in an $A \rightarrow B$ context. For example, if $C$ coincides with $A$, one cannot from the assertion that $A$ is $a$ at $t$ in an $A \rightarrow A \rightarrow B$ context infer a value for $A$ at $t$ in an $A \rightarrow B$ context. Nevertheless, various theories (or theory interpretations) can make claims about ontological properties of $C$ in an $A \rightarrow B$ context. They can, for instance, make assertions about the "true value" of $C$. It is obvious that such claims are absolutely untestable, since a test would involve measuring $C$, thereby landing in an $A \rightarrow C \rightarrow B$ context. This, however, does not imply that these claims have no meaning (for people other than strict empiricists, at least) or are not interesting.

In quantum mechanics, it cannot be maintained that $C$ has a true value (equal to one of its eigenvalues) revealed in and unaffected by a measurement. This is a direct consequence of the formalism and holds in any interpretation whatsoever. Indeed if $C$ had such a true value unaffected by measurement, the probability of $b$ conditional on $a$ would be given by

$$
P(b \mid a)=\sum_{i} P\left(b \mid c_{i}\right) P\left(c_{i} \mid a\right) .
$$

But in quantum mechanics $P(b \mid a)=|\langle b \mid a\rangle|^{2}$, with similar formulas for $P\left(b \mid c_{i}\right)$ and $P\left(c_{i} \mid a\right)$. These probabilities do not, in general, satisfy (2).

We should note that in an $A \rightarrow B$ context, quantum mechanics can consistently (although not compulsorily) be interpreted as asserting that the observable $A$ has a true value equal to $a$ between $t_{1}$ and $t_{2}$. That true value corresponds to Redhead's elements of reality. Now the Born and ABL rules are symmetric under the interchange of $a$ and $b$. This suggests also asserting that the observable $B$ has a true value equal to $b$ between $t_{1}$ and $t_{2}$. If both assertions are maintained, quantum mechanics must be an incomplete theory, because if observables $A$ and $B$ do not commute, eigenvectors of one are not in general eigenvectors of the other. We shall see, however, that there are good (though again not compelling) reasons for maintaining that $A$ has and $B$ does not have a true value between $t_{1}$ and $t_{2}$.

In hidden variable theories, nothing in principle prevents $C$, in an $A \rightarrow B$ context, from having a true value at all times between $t_{1}$ and $t_{2}$. Take for instance Bohmian mechanics. If $|a\rangle$ and $|b\rangle$ are not position eigenstates, then $A$ and $B$ do not commute with the position operator $X$. Yet the particle's 
position is well-defined at all times between $t_{1}$ and $t_{2}$. In an $A \rightarrow X \rightarrow B$ context, however, positions would be different. The measurement of $X$ at $t$ would from then on change the particle's wave function, and therefore its Bohmian trajectory.

Let us now examine how does Mohrhoff's interpretation of quantum mechanics fit in the present discussion of the ABL rule. In his exchange with R. E. Kastner [15, 25], Mohrhoff explains how to quantitatively define the counterfactual meaning of the ABL rule. To paraphrase him, saying that $P_{A B L}\left(c_{i}\right)$ is the objective probability with which $c_{i}$ would be obtained, given preparation $|a\rangle$ and measurement $b$ at $t_{1}$ and $t_{2}$, is "in all relevant aspects exactly the same as saying that" $P_{A B L}\left(c_{i}\right)$ is the subjective probability with which $c_{i}$ is obtained given the same outcomes at $t_{1}$ and $t_{2}$ [15, p. 868]. The relevant aspects here are the quantitative ones. As pointed out in section 2, however, it is clear that Mohrhoff has much more to say about the ontological consequences of the counterfactual meaning. The implications are that the observable $C$ categorically has no value between $t_{1}$ and $t_{2}$ in an $A \rightarrow B$ context. Recall that an observable has a value only when the value is ascertained by a fact. The subjective (uncontroversial) meaning of the ABL rule, which "in all relevant [quantitative] aspects" coincides with the objective (counterfactual) meaning, is held to entail an objective nonvaluedness of $C$ in the $A \rightarrow B$ context. It should be stressed once more that the nonvaluedness does not logically follow from the counterfactual meaning of the ABL rule. It is, nonetheless, a possible (and certainly a significant) interpretation based upon it.

The ABL rule, written as in (1), appears invariant under time reversal. Indeed let the signs of $t_{1}, t$, and $t_{2}$ be changed and let bras be transformed into kets under time reversal. Then the ABL rule is transformed into itself. But suppose the process of measurement, instead of occurring at a single instant, is spread over some interval of time. The time-reversal invariance of the ABL rule then depends on the time-reversal properties of the measurement process. This has been stressed by L. Vaidman [26] as far as the $C$ measurement goes. Similar considerations can be made for the initial preparation $(a)$ and final measurement $(b)$. In the case where the time reversal of preselection is postselection and where the $C$ measurement is time-reversal invariant, then the ABL rule is also time-reversal invariant. But this does not have to be so. Take for instance von Neumann's theory of measurement [11. In that approach, a measurement is an interaction followed by a collapse, a complex process that can be symbolized as $\operatorname{Int}(C) \rightarrow\left(C: c_{i}\right)$. The $A \rightarrow C \rightarrow B$ 
process can then schematically be represented as

$$
\operatorname{Int}(A) \rightarrow(A: a) \rightarrow \operatorname{Int}(C) \rightarrow\left(C: c_{i}\right) \rightarrow \operatorname{Int}(B) \rightarrow(B: b) .
$$

This is certainly not time-reversal invariant. For the purpose of computing probabilities of measurement results at time $t$, state $|a\rangle$ and state $|b\rangle$ are equally useful. Fot the purpose of making ontological statements, however, it is more natural in the absence of a $C$ measurement to hold that $|a\rangle$, rather than $|b\rangle$, is the intermediate state, since $|b\rangle$ obtains only after an intervening interaction.

In Mohrhoff's interpretation, however, the time-reversal invariance of the ABL rule can be held consistently. The reason is that in this framework, measurements should not be analyzed, as they are irreducible facts. This raises questions of its own, to which we shall come back later on.

\section{The quantum state}

I have already pointed out that Mohrhoff categorically denies that the "state vector" $|\psi(t)\rangle$ represents an evolving quantum state. "The idea that what by definition is a tool for assigning probabilities to possibilities also describes an actual state of affairs, is simply a category mistake." [14, p. 22] Most people will agree, so the argument goes, that $|\psi(t)\rangle$ represents a probability measure, that it yields the probability of various results on the condition that a measurement is performed. But if no measurement is performed, no observable has a value. A state and a probability measure are two entirely different objects. Identifying one with the other is therefore logically inconsistent.

I shall argue that such a strong claim is unfounded. Nevertheless, it is entirely consistent (though not compulsory) to deny the existence of an evolving quantum state. This is in fact an interpretative statement. Although it does bring substantial benefits, it also carries problems of its own.

The greatest benefit reaped in denying the existence of an evolving quantum state is that the ominous quantum measurement problem is, if not completely solved, at least considerably attenuated. In broad outline the measurement problem goes as follows. An observable $A$ with eigenvectors $\left|a_{i}\right\rangle$ and (say) nondegenerate eigenvalues $a_{i}$, pertaining to a quantum system $S$, is subject to a measurement by an apparatus $M$. The apparatus is also to be treated quantum mechanically. It starts in some initial state $\left|\alpha_{0}\right\rangle$. Suppose the quantum system is prepared in a state $\left|a_{j}\right\rangle$. A reliable apparatus should 
be built so that after suitable interaction between $S$ and $M$ (associated with a unitary operator $U$ ), the "pointer" of the apparatus shows a characteristic value $\alpha_{j}$, corresponding to a state $\left|\alpha_{j}\right\rangle$. Readings $\alpha_{i}$ and $\alpha_{j}(i \neq j)$ are unambiguous provided that $\left|\alpha_{i}\right\rangle$ and $\left|\alpha_{j}\right\rangle$ are macroscopically distinct. But now suppose that the quantum system is prepared in a nontrivial superposition $\sum_{k} c_{k}\left|a_{k}\right\rangle$ of eigenstates of $A$. Then the linearity of the evolution operator implies that after interaction, the joint quantum state of the system and apparatus is given by

$$
|\chi\rangle=\sum_{k} c_{k}\left|a_{k}\right\rangle \otimes\left|\alpha_{k}\right\rangle
$$

This result seems to be flatly ruled out by experiment, inasmuch as no apparatus is ever observed in a superposition of macroscopically distinct pointer states.

To derive this contradiction, it is crucial to view the state vector (41) as indeed representing a state, that is, an objectively existing state of affairs. Only then has the experimental observation any relevance in ruling it out. If instead $|\chi\rangle$ represents a probability measure for results $\alpha_{k}$ on the condition that one of them shows up, the contradiction is removed.

There still remains, however, what is known as the "pointer problem." In (4), the $\left|\alpha_{k}\right\rangle$ make up an orthonormal set of eigenvectors of an observable in the apparatus's Hilbert space, which may be denoted by $\mathcal{A}$. Let $\left\{\left|\beta_{l}\right\rangle\right\}$ be another orthonormal set, related to the first one by some unitary operator. The $\left|\beta_{l}\right\rangle$ can be thought of as eigenvectors of some (nonunique) operator $\mathcal{B}$. One can write

$$
|\chi\rangle=\sum_{l} c_{l}^{\prime}\left|b_{l}\right\rangle \otimes\left|\beta_{l}\right\rangle
$$

The $\left|b_{l}\right\rangle$ are called "relative states." [27] They are (in general nonorthogonal) linear combinations of the $\left|a_{k}\right\rangle$.

The pointer problem is encapsulated in the following question: What, from a fundamental point of view, makes vectors $\left|\alpha_{k}\right\rangle$ more relevant than vectors $\left|\beta_{l}\right\rangle$ ? Decoherence theory [4] attempts to answer the question by bringing up effects of the environment. Barring that, the $\left|\alpha_{k}\right\rangle$ are singled out by the following mathematical criterion: in general they are the only ones for which the relative states $\left|a_{k}\right\rangle$ are orthogonal ${ }^{5}$ and, therefore, are the eigenstates of a Hermitian operator.

\footnotetext{
${ }^{5}$ It is well-known that the biorthogonal decomposition (4) is unique if all $c_{k}$ have different norms.
} 
This criterion may be related to what Mohrhoff has in mind when he states that Born probabilities are conditional, among other things, on "the observable $[A]$ that is being measured." [14, p. 26] Whether this solves the pointer problem is, however, far from carrying consensus. Mohrhoff's interpretation does not seem to bring any additional insight to the issue.

To sum up, denying that the state vector represents an evolving quantum state considerably weakens the measurement problem, in a consistent way. Nevertheless, we shall see that from a logical point of view, this stand is by no means compelling.

It is true that a state description and a probability measure specify distinct classes of objects. But these classes are not mutually exclusive, anymore than in mathematics, for instance, the class of topological spaces excludes the class of groups. Perhaps one can argue that it is intuitively strange to endow a probability measure with properties of a state description. The converse, however, is not strange at all. Indeed there are good reasons to believe that if the state (i.e. the actual state of affairs) is known, predictions of some sort can be made as regards results of measurements. This is trivially true, for example, in classical mechanics. There the state is specified by a point in phase space at some time $t$. From this specification, a (trivial) probability measure can be defined on possible states at time $t^{\prime}$.

Nor does the requirement of collapse, in theories or interpretations in which it occurs, make the notion of an evolving state inconsistent. Clearly, if one is willing to contemplate deviations from Schrödinger evolution, numerous collapse models are possible that may have various degrees of likeliness. But even if one insists that the Schrödinger equation holds exactly, behavior akin to collapse is possible, as Bohmian mechanics clearly, and decoherence theory more controversially, illustrate.

I should point out that there are good (though again not compelling) reasons to view the state vector as specifying an evolving state. Let system $S$ be prepared at time $t_{1}$ in the state $\left|\psi\left(t_{1}\right)\right\rangle$. The preparation being ascertained by a fact, it is agreed that at time $t_{1}, S$ possesses the property associated with the projector $\left|\psi\left(t_{1}\right)\right\rangle\left\langle\psi\left(t_{1}\right)\right|$. Assume the system's Hamiltonian is known in the time interval $\left(t_{1}, t_{2}\right)$. Then in principle, the state vector $|\psi(t)\rangle$ is also known at any $t$ between $t_{1}$ and $t_{2}$. Hence one can predict with certainty that a measurement, at time $t$, of the property associated with the projector $P_{\psi(t)}=$ $|\psi(t)\rangle\langle\psi(t)|$ will yield the value one. In Redhead's language, $P_{\psi(t)}=1$ is a (time-dependent) element of reality. This possibility of correctly predicting the value of a time-dependent dynamical variable motivates the association 
of the state vector with an actual state of affairs. ${ }^{6}$

Mohrhoff rightly points out that no detector is $100 \%$ efficient. The same applies to preparation devices. A preparation device may not always prepare anything, or it may prepare something slightly different from what is intended. Thus in the real world one may be wrong when one assigns an element of reality. Yet one can consistently maintain that there is an element of reality in, say, $99 \%$ of cases. In investigating the extent to which facts do or do not have causes, it may help to remember that the inefficiency of preparation devices is irrelevant to the existence or nonexistence of elements of reality.

\section{Macroscopic objects and facts}

In Mohrhoff's interpretation, the formalism of quantum mechanics is taken to hold exactly. Hence it should apply to everything, including macroscopic objects.

How are macroscopic objects to be defined? We have seen that no object (whatever its size) has a position unless that position is indicated by a fact. A fact will occur in relation with a detector that can monitor the object's position up to a certain precision. Now the bigger an object is, the slower the wave packet associated with its center-of-mass position will spread (the spreading goes roughly as $m^{-1}$, where $m$ is the object's mass). Hence for large objects, center-of-mass positions (or indeed center-of-mass positions of their parts) have very little time to become appreciably fuzzy between two successive position-indicating facts. That very small fuzziness, to show itself, requires a much bigger detector that can monitor positions with very high precision. The bigger detector's position still has a residual, exceedingly small fuzziness, which however can only be revealed by still another, this time exceedingly large, detector. Clearly, in the actual world, this process cannot go on forever. Detectors cannot be arbitrarily large, if only to be stable against gravitational collapse. The upshot is that there are objects so

\footnotetext{
6 "As my aim here is to make sense of standard quantum mechanics, I take the nonexistence of hidden variables for granted." [14 p. 16] If elements of reality are reckoned to be outside the formalism of quantum mechanics, this statement eliminates them tautologically. What is really significant, I believe, is that elements of reality and hidden variables can be introduced while in no way changing the Schrödinger equation, the association of measurement results with eigenvalues of Hermitian operators, or the Born rule.
} 
large that no detector exists to reveal the fuzziness of their position. These are, by definition, macroscopic objects.

It is experimentally well established that, with one type of exception, bulk properties of macroscopic objects follow the laws of classical mechanics. The exception occurs, of course, when the macroscopic object is used as a pointer to indicate the unpredictable value of a microscopic object's observable. But barring that, classical mechanics provides an entirely adequate description of things like footballs and planets. Now these are also subject to the laws of quantum mechanics, inasmuch as the latter apply to everything. Can the two laws be made consistent? There are strong (although not yet entirely compelling, see [6]) indications that if a macroscopic object is treated quantum mechanically, then quantum observables can be defined whose probabilistic behavior follows very closely the classical behavior of associated classical observables. Is this enough to conclude that classical mechanics, now understood as the causal description of the succession of facts, is reducible to quantum mechanics? Not so for Mohrhoff.

The reason is that quantum mechanics never predicts the occurrence of a fact. On the basis of facts, it predicts the probability of various results if an additional fact occurs. But then, what is the precise nature of a fact?

Mohrhoff does give some answers to this question. He points out that facts play in quantum mechanics a role similar to initial conditions in classical mechanics. "While in classical physics actuality attaches itself to a nomologically possible world trivially through the initial conditions, in quantum physics it 'pops up' unpredictably and inexplicably with every propertydefining fact." [12, p. 729] As initial conditions are always assumed and never explained, so should facts be. "[T]he existence of facts - the factuality of events or states of affairs - cannot be accounted for, anymore than we can explain why there is anything at all, rather than nothing." [16, p. 221]

It would seem that in some circumstances, the occurrence of a fact can be predicted, if not through quantum mechanics, at least empirically. Take for instance a Geiger counter together with a device that can prepare a charged particle with a position and speed as well-defined as Heisenberg's principle allows. At $t_{1}$ the particle is emitted towards the counter with a speed set so that it arrives at $t_{2}$. Here $t_{1}$ and $t_{2}$ are defined in terms of nearby macroscopic clocks. With this setup one can predict, with a high degree of probability, that the counter will click at $t_{2}$.

It turns out, however, that things are more complicated. The situation just described does not evince a unique fact. Before and after the click one 
can, within the finite time differentiation allowed by available clocks, repeatedly and positively ascertain that there is no click. All these "nonclicks" are facts. When the charged particle enters the Geiger counter, the particle's and counter's wave functions become entangled, and there is a growing probability for click rather than nonclick. I agree with Mohrhoff that standard quantum mechanics is of no help in predicting when precisely will the click occur.

It is entirely consistent to view the occurrence of facts as uncaused and unpredictable. From an epistemological point of view, however, it is risky. Classical mechanics does not explain initial conditions, but it explains everything else. That is, the totality of the world at one instant is unexplained, but the totality of the world at all other instants is explained. Mohrhoff tells us that in quantum mechanics, all the facts that constantly betoken positions of macroscopic objects should be taken as unexplainable. It seems that the unexplained here far exceeds what it is in classical mechanics. Inevitably, people will look for regularities in the occurrence of facts (beyond the consistency of classical trajectories with quantum-mechanical probabilities). In a sense, spontaneous localization theory [7] may be viewed as doing just that. It may or may not be successful. But, as pointed out in Ref. [28], any theory that would correctly account for the occurrence of facts would, other things being equal, have a head start over one that does not.

Other properties of facts may be subject to experimental investigation and, therefore, call for theoretical analysis. A fact occurs every time when, for instance, a detector clicks or a pointer deflects. It is crucial to observe that the fact does not coincide with the pointer's position. [14, p. 31] The latter is, ultimately, a quantum observable subject to a fuzziness that is just too small for a suitable detector to evince it. But the former is not a quantum observable (it allows assigning values to quantum observables), and it is not subject to fuzziness. Now it will presumably be agreed that a $1 \mathrm{~kg}$ detector can register a fact. What about smaller detectors? Can a $1 \mathrm{~g}$ detector register a fact, or a $1 \mu \mathrm{g}$ detector, or a smaller one? Is a detector required to have some minimum mass to be able to work as a detector? If the answer is yes, that mass ought to be open to investigation.

But perhaps the answer is no. Maybe even atoms or elementary particles can work as detectors. Then another question comes up. Consider a hydrogen atom in a $2 p$ state, confined in a high vacuum. In a split second the atom is very likely to emit a $1216 \AA$ photon and fall to the ground state. Perhaps one will maintain that when the photon has gone far enough, a fact has 
occurred. But then the question is, When did the fact occur? It won't help to say that time for the atom is not defined outside the context of the fact, for the occurrence can be referred to a nearby macroscopic clock. A matter of fact "is something that cannot be undone or 'erased'." [13, p. 18] If it is agreed that the fact has already occurred at $t=1 \mathrm{~s}$ (as shown by the macroscopic clock), it must have occurred at some instant between $t=0$ and $t=1 \mathrm{~s}$. That instant is defined as the one starting from which it is impossible to undo or erase. Some way or other, it ought to be open to experimental investigation.

\section{Discussion}

The interpretation of quantum mechanics proposed by U. Mohrhoff bears resemblance to the Copenhagen interpretation. It takes quantum mechanics to be fundamental and complete, and it requires the validity of classical mechanics for its formulation. In both the state vector is a tool for calculating probabilities. Yet Mohrhoff's interpretation goes beyond the Copenhagen interpretation in several ways. It applies not only to measurements in the strict sense, but to all property-indicating facts. Moreover, it does not share the Copenhagen interpretation's strict instrumentalism. Far from remaining silent about the behavior of quantum observables in between measurements, it explicitly asserts their fuzziness or nonvaluedness or, equivalently, the meaninglessness of their having a value. That nonvaluedness applies most importantly to space and time, which are undefined outside the context of measurements or facts. "The seemingly intractable problem of understanding quantum mechanics is a consequence of our dogged insistence on obtruding onto the world, not a spatiotemporal framework, but a spatiotemporal framework that is more detailed than the world." [12, p. 742]

Probability statements have a very specific meaning in Mohrhoff's interpretation. Objective statements apply counterfactually to measurements that are not performed. Subjective statements are rational guesses made on the basis of incomplete information, in particular if the result of an actually performed measurement is not known. The "state vector" is viewed strictly as a probability measure, and emphatically not as representing an evolving quantum state. This goes a long way towards solving the quantum measurement problem. It remains, however, an interpretational statement, not logically implied by the formalism of quantum mechanics. The same 
holds for the rejection of elements of reality and the assertion of an objective nonvaluedness in between measurements or facts.

Mohrhoff presents a view of macroscopic objects that carries much appeal. To them quantum mechanics applies universally and exactly. Their positions are quantum observables, qualitatively subject to fuzziness just like observables associated with microscopic objects. Quantitatively, however, the fuzziness is exceedingly small, in effect so small that there are no detectors large enough to evince it. That view of macroscopic objects should, I believe, be investigated further. The notion that an object's position always shows up through another object's fuzzy position may entail consequences otherwise not so easily uncovered.

Outside the explanatory scheme of quantum mechanics are facts, which are uncaused and unpredictable. Quantum mechanics predicts the probability of measurement results on the condition that a fact occurs, but it says nothing about the occurrence itself. This raises questions about the nature of facts and the extent to which they can be experimentally investigated as well as theoretically analyzed. These questions, although framed in a different setup, are related to the ones often asked about the frontier between the quantum and the classical.

In the semantic view of scientific theories [8], a theory is identified with the class of its models or interpretations. Each model answers the question, How can the world be the way the theory says it is? The models collectively give meaning to the theory. In the interpretation he has put forth, Mohrhoff has shown us a thought-provoking and original view of the way that, according to quantum mechanics, the world can be.

\section{Acknowledgments}

I am grateful to Ulrich Mohrhoff for a stimulating exchange of views.

\section{References}

[1] D. Bohm, "A suggested interpretation of the quantum theory in terms of 'hidden' variables (I and II)," Phys. Rev. 85, 166-193 (1952).

[2] D. Bohm and B. J. Hiley, The Undivided Universe (Routledge, London, 1993). 
[3] P. R. Holland, The Quantum Theory of Motion (Cambridge University Press, Cambridge, 1993).

[4] W. H. Zurek, "Decoherence and the transition from quantum to classical," Phys. Today 44, No. 10, 36-44 (1991).

[5] R. B. Griffiths, "Consistent histories and the interpretation of quantum mechanics," J. Stat. Phys. 36, 219-272 (1984).

[6] R. Omnès, The Interpretation of Quantum Mechanics (Princeton University Press, Princeton, 1994).

[7] G. C. Ghirardi, P. Pearle, and A. Rimini, "Markov processes in Hilbert space and continuous spontaneous localization of systems of identical particles," Phys. Rev. A 42, 78-89 (1990).

[8] B. C. van Fraassen, Quantum Mechanics: An Empiricist View (Clarendon Press, Oxford, 1991).

[9] P. E. Vermaas, A Philosopher's Understanding of Quantum Mechanics. Possibilities and Impossibilities of a Modal Interpretation. (Cambridge University Press, Cambridge, 1999).

[10] B. d'Espagnat, Veiled Reality. An Analysis of Present-Day Quantum Mechanical Concepts (Addison-Wesley, Reading, 1995).

[11] J. von Neumann, Mathematical Foundations of Quantum Mechanics (Princeton University Press, Princeton, 1955).

[12] U. Mohrhoff, "What quantum mechanics is trying to tell us," Am. J. Phys. 68, 728-745 (2000).

[13] U. Mohrhoff, "Quantum mechanics and elements of reality," e-print quant-ph/9904081 (1999).

[14] U. Mohrhoff, "The one, the many, and the quantum," e-print quant-ph/0005110 (2000).

[15] U. Mohrhoff, "Objective probabilities, quantum counterfactuals, and the ABL rule - A response to R. E. Kastner," Am. J. Phys. 69, 864-873 (2001). 
[16] U. Mohrhoff, "The world according to quantum mechanics (or the 18 errors of Henry P. Stapp)," Found. Phys. 32, 217-254 (2002).

[17] U. Mohrhoff, "Making sense of a world of clicks," Found. Phys. 32, 1295-1311 (2002).

[18] N. D. Mermin, "What is quantum mechanics trying to tell us?," Am. J. Phys. 66, 753-767 (1998).

[19] K. R. Popper, "Quantum mechanics without 'the observer'," in Quantum Theory and Reality, M. Bunge, ed. (Springer, New York, 1967), pp. $7-44$.

[20] Y. Aharonov, P. G. Bergmann, and J. L. Lebowitz, "Time symmetry in the quantum process of measurement," Phys. Rev. 134B, 1410-1416 (1964).

[21] A. Einstein, B. Podolsky, and N. Rosen, "Can quantum-mechanical description of physical reality be considered complete?," Phys. Rev. 47, 777-780 (1935).

[22] M. Redhead, Incompleteness, Nonlocality, and Realism (Clarendon Press, Oxford, 1987).

[23] W. D. Sharp and N. Shanks, "The rise and fall of time-symmetrized quantum mechanics," Philos. Sci. 60, 488-499 (1993).

[24] D. Z. Albert, Y. Aharonov, and S. D'Amato, "Curious new statistical prediction of quantum mechanics," Phys. Rev. Lett. 54, 5-7 (1985).

[25] R. E. Kastner, "Comment on "What quantum mechanics is trying to tell us,' by Ulrich Mohrhoff," Am. J. Phys. 69, 860-863 (2001).

[26] L. Vaidman, "Time-symmetrized counterfactuals in quantum theory," e-print quant-ph/9807075 (1998).

[27] H. Everett III, "'Relative state' formulation of quantum mechanics," Rev. Mod. Phys. 29, 454-462 (1957).

[28] H. P. Stapp, "The 18-fold way," Found. Phys. 32, 255-266 (2002) 